Research article

\title{
Ethnomedicinal aspects of climbing plants of Palpa district, Nepal
}

\author{
Anant Gopal Singh ${ }^{1}$ and Akhilesh Kumar $^{2} *$ \\ ${ }^{1}$ Department of Botany, Tribhuvan University, Butwal Multiple Campus, Butwal, Nepal \\ ${ }^{2}$ Department of Botany, Dayalbagh Educational Institute (Deemed University), Agra, Uttar Pradesh, India
}

*Corresponding Author: akhilpbh@ @rediffmail.com

[Accepted: 23 July 2017]

\begin{abstract}
Climbers are those plants which germinate on land and grow by adhering to other plants to attain great stature. Climbers are different in their mechanical characters, well adapted to climb on support like large trees, hedgerows or rocks by twining their stems, some climbers climb on support by the way of adventitious clinging roots, with twining petioles or by using tendrils. A total of 51 climbing plants species belonging to 40 genera and 22 families used in folk medicine have been documented from different VDCs of Palpa district.
\end{abstract}

Keywords: Climber - Clinging roots - Tendrils - Ethnomedicine - Liane.

[Cite as: Singh AG \& Kumar A (2017) Ethnomedicinal aspects of climbing plants of Palpa district, Nepal. Tropical Plant Research 4(2): 307-313]

\section{INTRODUCTION}

Climbing plants are one of the most interesting group but a much-neglected group of plants. A climbing habit has evolved independently in several plant families, using many different climbing methods because of their weak stem. Climbers are different in their mechanical characters (Isnard et al. 2009), well adapted to climb on support like large trees, hedgerows or rocks. Some climbers climb by twining their stems around a support another climb by way of adventitious clinging roots, with twining petioles or using tendrils which can be specialized shoots, leaves or even inflorescence.

Climbers are the plants that germinate on the floor and grow for part of their life by winding ground, anchoring or adhering to other plants (Jongkind \& Hawthrone 2005), to attain great stature (Swaine et al. 2005). They comprise $7 \%$ and $20 \%$ of regional flora in temperate and tropical forest respectively (Gentry 1991) and thus become an important constituent of an area. Climbers are not only the important components of plant diversity but, are also valuable for their medicinal uses, nutrient cycling etc. (Schnitzer \& Bongers 2002). The climbers are rooted in the ground but need support for their weak stems (Richards 1952). In nature, climbers generally use plants growing nearby as external support media to ascend (den Dubbelden \& Oosterbeek 1995). Schimper (1903) classified climbers into scrambles, root climbers, twiners and tendril climbers. The term 'vine' and liane are generally used as synonymous for climber and woody climbers respectively (Kelly 1985).

Today the climbers are an important constituent of non-timber forest products (NTFPs) According to the best of our knowledge there is no comprehensive study assessing the role of climbers in Nepalese forests for healthcare management and economic subsistence of local people. Keeping above view in mind present study was proposed to document the ethnomedicinal uses of climbers for the first time in the Palpa district of Western Nepal.

\section{MATERIALS AND METHODS}

Study Area

Palpa district is a part of Lumbini Zone, is one of the seventy-five districts of Nepal, landlocked country of South Asia, is located at $27^{\circ} 34^{\prime \prime}$ to $27^{\circ} 54^{\prime \prime} \mathrm{N}$ and $83^{\circ} 15^{\prime \prime}$ to $84^{\circ} 22^{\prime \prime} \mathrm{E}$ with an altitude ranging from $152 \mathrm{~m}$ to $1936 \mathrm{~m}$ above the sea level. It has unique physiography due to Churia and Mahabharat ranges. The district, with Tansen as its headquarters, covers an area of $1,373 \mathrm{Km}^{2}$ and has a population of 2, 61,180 of which male and female proportions are 1, 15,840 and 1, 45,340 respectively (CBS 2011). Palpa district is divided into two municipalities i.e. Tansen and Rampur and 61 VDCs. It is bounded by Gulmi and Arghakhanchi districts from West; Gulmi, Syangja, and Tanhun district from North; Nawalparasi and Tanhun from East and Rupandehi and 
Nawalparasi from South (Fig. 1). Palpa district is located in hilly region of Nepal. The average temperature of the district is maximum $32^{\circ} \mathrm{C}$ and minimum $4^{\circ} \mathrm{C}$ (DDC 2009) with dry winter and wet summer. The average annual rainfall is around $2006 \mathrm{~mm}$, with a fluctuating pattern for total of 29 years between 1985 and 2013 A.D., (DCEP 2016). The monsoon starts from June and pronounce rainfall occurs during June to mid-September. Palpa district of Western Nepal is inhabited by many ethnic communities of which $50 \%$ or majority is comprised by Magars and rest by Brahmin, Newar, Chhetri, Gurung, Kumal, Sarki, etc.

Geographically Palpa district is divided into mid-mountain hill region (82\%) and Chure hill region (18\%). Total forest in Palpa district is 67607 ha, the land area used for agriculture is 44,332 ha, shrubs area is 23,736 ha with 538 ha area of water bodies and 70 ha of barren land. The vegetation of the Palpa district is dominated on the southern side by lower tropical Sal (Shorea robusta Gaertn.) and mixed broad leaves forest. Terminalia alata Heyne ex Roth, T. chebula Retz., T. bellirica (Gaertn.) Roxb., Dalbergia sissoo DC., Ficus semicordata Buch.-Ham. ex Sm., Castanopsis indica (Roxb. ex Lindl.) A.DC., Berberis asiatica Roxb. ex DC., Zanthoxylum armatum DC., Albizia species, Schima wallichii (DC.) Korth., and Pinus roxburghii Sarg. etc. are also found dominated in the upper and mid belt of the district.

Palpa district of Western Nepal is selected for research activities because this district is potential in terms of ethnomedicine. Little works have been carried out by some workers (Shrestha 1985, Mahato 1998, Mahato \& Chaudhary 2003, Singh et al. 2011) but no any work has been done on climbing plants.

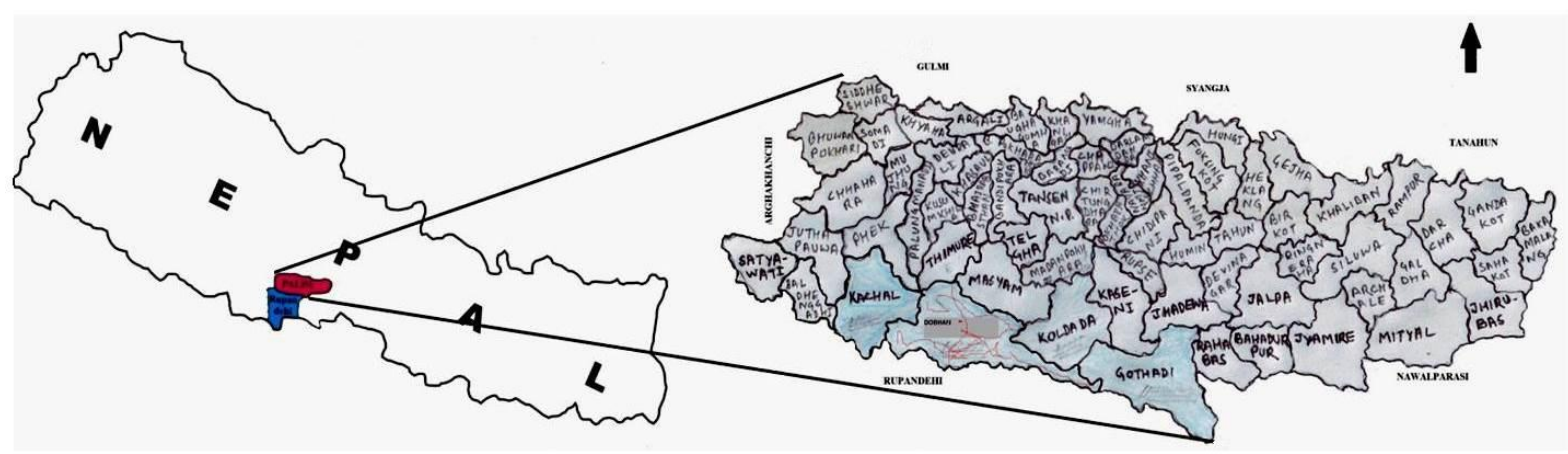

Figure 1. Location map of study site: Palpa district.

\section{Data Collection}

A preliminary survey was done in different VDCs like Kachal, Dobhan, Koldanda, Gothadi, Rahabas, and Jyamire to get information about the study area along with local persons and knowledgeable peoples. The area was visited three times in different seasons (summer, monsoon, and winter) to avail most of the plant resources in their flowering condition for this purpose. The study was conducted from October 2014 to August 2015. The methodology was based on interviews using checklist and questionnaire of information.

Plants collected from study area were identified by using previous literature (Hooker 1872-1897, Bailey 1949, DMP 1969, 1970, 1984, 1986, Polunin \& Stainton 1984, Mc Crackers \& Shrestha 1992, Stainton 1997, Chaudhary 1998, Manandhar 2002). Photographs were taken of the plants' habit in the flowering conditions, abundant plants were taken to prepare herbarium sheets. Herbarium specimens and photographs were identified by taxonomists and finally deposited in the Herbarium of the Department of Botany, Butwal Multiple Campus, T.U., Butwal, Nepal for future use. The questionnaire was prepared in terms of local language following Martin's (1995) manual. The Scientific name of the collected climbing plants were provided by Hara et al. (1978, 1979, 1982) and Press et al. (2000) have been adopted.

\section{RESULTS AND DISCUSSION}

Survey of palpa district revealed that 51 climbing plants species belonging to 40 genera and 22 families are used in folk medicine by the inhabitants of different VDCs like Kachal, Dobhan, Koldanda, Gothadi, Rahabas, and Jyamire of Palpa district. Out of these climbing plants 38 plants belong to Dicots, 12 belong to Monocots and 1 belongs to Fern. The highest number of climbing plants belong to family Cucurbitaceae (12 species) followed by Fabaceae ( 8 species), Convolvulaceae, Dioscoreaceae, Piperaceae (3 species in each), Vitaceae, Menispermiaceae, Asclepiadaceae, Liliaceae, Smilaceae (2 species in each) etc. Singh (2016) described ethnobotany of 18 climbers of Paras district of Nepal. Out of these, 15 namely climbers Abrus precatorius, Acacia rugata, Aristolochia indica, Bauhinia vahlii, Cissampelos pareira, Cuscuta reflexa, Dioscorea bulbifera, 

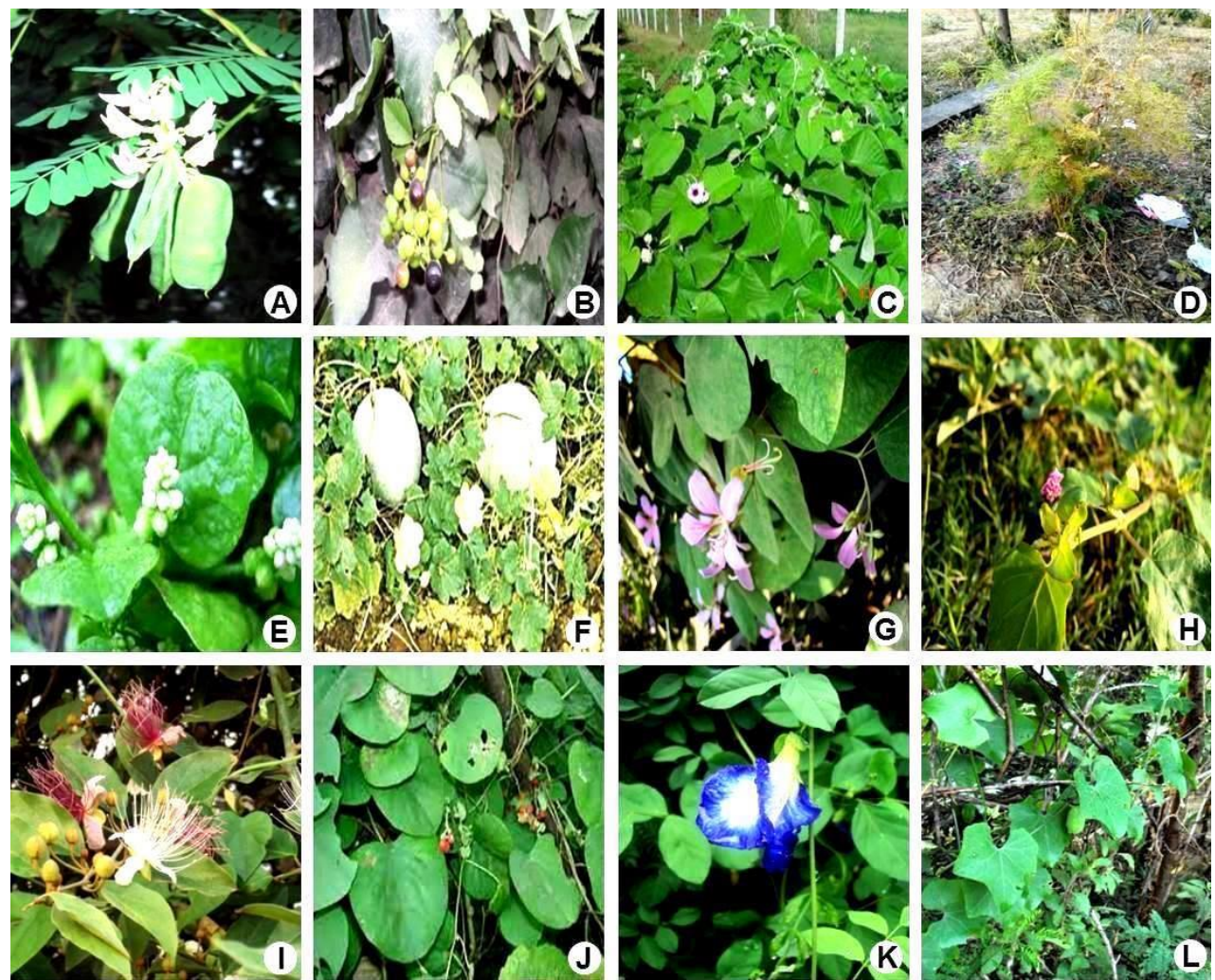

Figure 2. Some ethnomedicinally important climbers: A, Abrus precatorius L.; B, Ampelocissus barbata (Wall.) Planch; C, Argyreia nervosa (Burm.f.) Bojer; D, Asparagus racemopsus Willd; E, Basella alba L.; F, Benincasa hispida (Thumb.) Cogn.; G, Bauhinia vahlii Wight \& Arn; H, Boerhaavia difusa L.; I, Capparis zeylanica L.; J, Cissampelos pariera L.; K, Clitorea ternatea L.; L, Coccinia grandis (L.) Voigt.
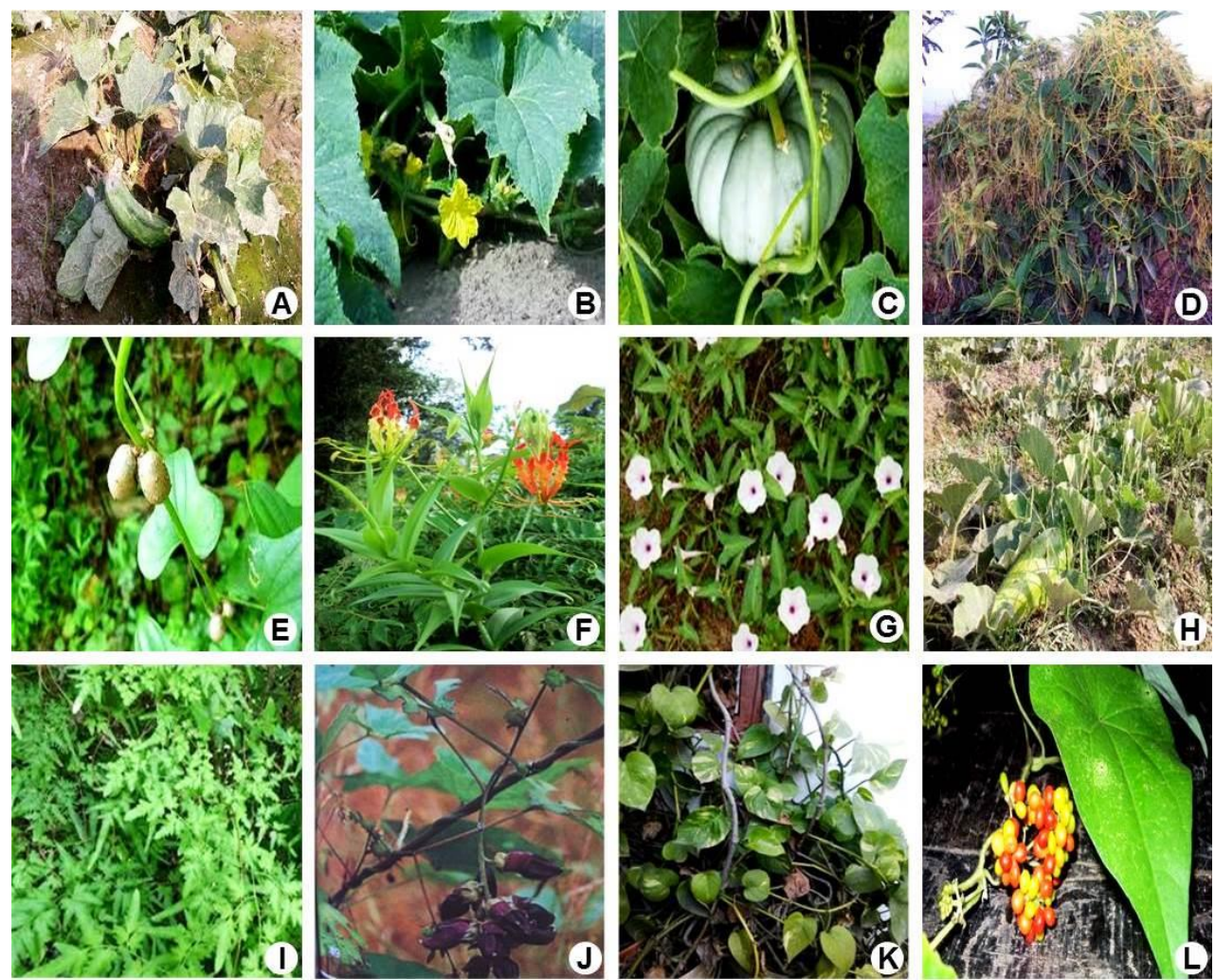

Figure 3. Some ethnomedicinally important climbers: A, Cucumis sativus L.; B, Cucurbita maxima Duch. ex Lam.; C, Cucurbita pepo L.; D, Cuscuta reflexa Roxb.; E, Dioscorea deltoidea Wall. ex Griseb.; F, Gloriosa superba L.; G, Ipomea aquatic Forssk.; H, Lagenaria sineraria (Molina) Standl.; I, Lygodium japonicum (Thunb.) Sw.; J, Mucuna pruriens (L.) DC.; L, Pothos scandens L.; M, Tinospora cordifolia (Willd.) Miers. 
D. deltoidea, Gymnema sylveste, Lygodium japonicum, Mucuna pruriens, Passiflora edulis, Quisqualis indica, smilax aspera and Tinospora cordifolia were recorded during the present study. Climbing plants used in folk medicine are enumerated in table 1 arranged in alphabetical order by their botanical name along with their family, local name and ethnomedicinal uses. The photographs of some of these plants have also been provided (Fig. 2 \& 3). These plants with high medicinal values are suggested here for the biological screening to develop the valuable pharmacological products.

Table 1. Ethnomedicinal usages of climbing plants of Palpa district, Nepal.

\begin{tabular}{lll}
\hline Botanical name and Family & Local name & Ethnomedicinal uses \\
\hline 1. Abrus precatorius L. & Ratti Gedi & Bronchitis, diuretic, tonic, abortifacient, boils, eczema, \\
Fabaceae & & leucoderma, jaundice, back bone pain and gonorrhoea. \\
2. Accacia rugata (Lam.) & Sikakai & Fruit used as detergent, young shoot cooked as vegetable. \\
Voigt & &
\end{tabular}

Voigt

Fabaceae

3. Ampelocissus barbat

Jarilo Lahara

Bone fracture, skin diseases.

(Wall.) Planch

Vitaceae

\section{Asparagus racemosus Kurilo}

Willd.

Liliaceae

5. Argyreia nervosa Samundra phal

(Burm.f.) Bojer

Convolvulaceae

6. Aristolochia indica L.

Aristolochiaceae

7. Basella alba $\mathrm{L}$.

Basellaceae

8. Bauhinia vahlii Wight \& Bhorla

Arn.

Fabaceae

9. Benincasa hispida

(Thumb.) Cogn.

Cucurbitaceae

10. Boerhavia diffusa L. Punarnawa

Nyctaginaceae

\section{Calamus erectus Roxb.}

Arecaceae

12. Capparis zeylanica $\mathrm{L}$.

Capparaceae

13. Cissampelos pariera L. Batulpate

Menispermiaceae

14. Cissus quadriangularis L. Hathzode Vitaceae

\section{Clinopodium umbrosum}

(M. Bieb) C. Koch.

Lamiaceae

16. Clitoria ternatea $\mathrm{L}$.

Fabaceae

17. Coccinia grandis (L.)

Voigt.

Kubindo

Kukurkande

Cucurbitaceae

Aparajita

Golkankri/

Kunaroo
Aphrodisiac, galactagogue, urinary trouble, nervous debility, bronchitis, dysentery \& throat infection.

Aphrodisiac, dyspepsia, colic, anaemia, anti-diabetic, cardiac debility, nervous debility, weakness \& syphilis.

Diuretic, stimulant, anti-arthritic, anti-diabetic, blood pressure, emetic, abortifacient \& emmenogogue Demulcent, insomnia, dermatitis, diuretic, \& laxative

Aphrodisiac, diarrhoea, malarial fever, tonic, boils, pimples, blisters \& blood dysentery.

Cooling, styptic (stop bleeding), laxative, diuretic, aphrodisiac, \& anthelmintic.

Pimples, scabies, cuts, wounds, stomachache, cardiac disorders, jaundice, anemia, constipation, cough, inflammations and bronchitis.

Anti-diabetic, dyspepsia, stomach problems, eczema \& wounds

Diuretic, resolvent, antipyretic, anti-arthritic, toothache, jaundice and spleen problems.

Diuretic, diarrhoea, dysentery, dyspepsia, malarial fever, pulmonary disease, piles, cough, antipyretic, cut, wounds, burns \& ring worm.

Boils, ulcers, rheumatism.

Suparnasa/ Birajor Cuts, burns, wounds
Leucoderma, liver disease, nervous tonic, abdominal pain.

Cataract, anti-diabetic, carminative, antipyretic, hepatoprotective 


\begin{tabular}{|c|c|c|}
\hline $\begin{array}{l}\text { 18. Cucumis sativus L. } \\
\text { Cucurbitaceae }\end{array}$ & Kankro & Galactagogue, refrigerant, diuretic. \\
\hline $\begin{array}{l}\text { 19. Cucurbita maxima Duch. } \\
\text { ex Lam. } \\
\text { Cucurbitaceae }\end{array}$ & Pharsi & Refrigerant, diuretic, neuralgia. \\
\hline $\begin{array}{l}\text { 20. Cucurbita pepo L. } \\
\text { Cucurbitaceae }\end{array}$ & Pharsi & $\begin{array}{l}\text { Anthelmintic, anti-inflammatory, kidney stones, urinary } \\
\text { tract infection. }\end{array}$ \\
\hline 21. Cuscuta reflexa Roxb. & Akashbeli/ & Jaundice, constipation, bronchitis, fever, \\
\hline Cuscutaceae & Amarbel & $\begin{array}{l}\text { antihypertensive, cardio-tonic, emetic, antiviral \& } \\
\text { antibacterial. }\end{array}$ \\
\hline $\begin{array}{l}\text { 22. Dioscorea alata L. } \\
\text { Dioscoreaceae }\end{array}$ & Ghar tarul & $\begin{array}{l}\text { Wounds, leprosy, gonorrhoea, blood pressure \& skin } \\
\text { diseases. }\end{array}$ \\
\hline $\begin{array}{l}\text { 23. Dioscorea bulbifera L. } \\
\text { Dioscoreaceae }\end{array}$ & Githa/ Ban tarul & $\begin{array}{l}\text { Piles, leprosy, asthma, cough, cold, tuberculosis, } \\
\text { contraceptive, constipation, indigestion, dysentery, syphilis, } \\
\text { cardiac debility, aphrodisiac, refrigerant, pulmonary } \\
\text { disease and ulcers. }\end{array}$ \\
\hline $\begin{array}{l}\text { 24. Dioscorea deltoidea } \\
\text { Wall. ex Griseb. } \\
\text { Dioscoreaceae }\end{array}$ & $\begin{array}{l}\text { Kukur tarul/ } \\
\text { Bhyakur }\end{array}$ & Stomachache, kill body lice, wash clothes. \\
\hline $\begin{array}{l}\text { 25. Dolichus lablab L. } \\
\text { Fabaceae }\end{array}$ & Rajsimi & $\begin{array}{l}\text { Emmenagogue, febrifuge, stomachic, anti-spasmodic, } \\
\text { aphrodisiac. }\end{array}$ \\
\hline $\begin{array}{l}\text { 26. Entada phaseoloides (L.) } \\
\text { Merr. } \\
\text { Fabaceae }\end{array}$ & Pangra & Epilepsy, constipation, anthelmintic \\
\hline $\begin{array}{l}\text { 27. Gloriosa superba L. } \\
\text { Liliaceae }\end{array}$ & Karihari & Stomachic, anthelmintic, dermatitis \& abortifacient. \\
\hline $\begin{array}{l}\text { 28. Gymnema sylvestris } \\
\text { (Retz.) R.Br. } \\
\text { Asclepiadaceae }\end{array}$ & $\begin{array}{l}\text { Gudmar/ } \\
\text { Madhunasini }\end{array}$ & Anti-diabetic, malarial fever, cough, cold, jaundice. \\
\hline $\begin{array}{l}\text { 29. Ipomea aquatic Forssk. } \\
\text { Convolvulaceae }\end{array}$ & $\begin{array}{l}\text { Laharo pani sag/ } \\
\text { Kerunga sag }\end{array}$ & $\begin{array}{l}\text { Emetic, purgative, gastric troubles, nervous and general } \\
\text { debility. Tender shoot used as green vegetable. }\end{array}$ \\
\hline $\begin{array}{l}\text { 30. Ipomea batatas (L.) Lam. } \\
\text { Convolvulaceae }\end{array}$ & Sakharakand & Refrigerant, laxative, aphrodisiac, diuretic \& tonic. \\
\hline $\begin{array}{l}\text { 31. Lagenaria sineraria } \\
\text { (Molina) Standl. } \\
\text { Cucurbitaceae }\end{array}$ & Lauka & $\begin{array}{l}\text { Anti-inflammatory, refrigerant, expectorant, purgative, } \\
\text { hepato-protective, emetic \& diuretic }\end{array}$ \\
\hline $\begin{array}{l}\text { 32. Luffa acutangula (L.) } \\
\text { Roxb. } \\
\text { Cucurbitaceae }\end{array}$ & $\begin{array}{l}\text { Pate Ghiroula/ } \\
\text { Taroi }\end{array}$ & $\begin{array}{l}\text { Skin diseases, demulcent, diuretic, tonic, nutritive, } \\
\text { purgative, emetic \& expectorant. }\end{array}$ \\
\hline $\begin{array}{l}\text { 33. Luffa cylindrica (L.) } \\
\text { Roem. } \\
\text { Cucurbitaceae }\end{array}$ & $\begin{array}{l}\text { Ghiu Taroi/ } \\
\text { Ghrimala }\end{array}$ & $\begin{array}{l}\text { Diuretic, emollient, laxative, expectorant, tonic, } \\
\text { anthelmintic, galactagogue, fever \& bronchitis. }\end{array}$ \\
\hline $\begin{array}{l}\text { 34. Lygodium japonicum } \\
\text { (Thunb.) Sw. } \\
\text { Lygodiaceae }\end{array}$ & Janai Lahara & Expectorant, diuretic, cough, cold, fever, anti-arthritic. \\
\hline $\begin{array}{l}\text { 35. Marsdenia roylei Wight. } \\
\text { Asclepiadaceae }\end{array}$ & Baahuni Lahara & Stomachache, purgative, gonorrhoea. \\
\hline $\begin{array}{l}\text { 36. Momordica charantia L. } \\
\text { Cucurbitaceae }\end{array}$ & Tite Karela & $\begin{array}{l}\text { Peptic ulcer, anti-diabetic, rheumatism. Anthelmintic, } \\
\text { antipyretic, purgative \& carminative. }\end{array}$ \\
\hline $\begin{array}{l}\text { 37. Momordica dioica Roxb. } \\
\text { ex Willd. } \\
\text { Cucurbitaceae }\end{array}$ & Bankarela & Rheumatism, anti-diabetic \\
\hline
\end{tabular}




\begin{tabular}{|c|c|c|}
\hline $\begin{array}{l}\text { 38. Mucuna monosperma } \\
\text { DC. } \\
\text { Fabaceae }\end{array}$ & Baldhengra & Expectorant, cough, asthma \\
\hline $\begin{array}{l}\text { 39. Mucuna pruriens (L.) } \\
\text { DC. Fabaceae. }\end{array}$ & Kauso & $\begin{array}{l}\text { Anthelmintic, aphrodisiac, kidney problems, uterine } \\
\text { trouble \& general debility. }\end{array}$ \\
\hline $\begin{array}{l}\text { 40. Paederia foetida } \mathrm{L} \text {. } \\
\text { Rubiaceae }\end{array}$ & $\begin{array}{l}\text { Bari Lahara/ } \\
\text { Gandha Prasarini }\end{array}$ & $\begin{array}{l}\text { Anti-arthritic, colic, flatulence, tonic, astringent, nervous } \\
\text { tonic, gastro protective, antioxidant } \& \text { anti-inflammatory. }\end{array}$ \\
\hline $\begin{array}{l}\text { 41. Passiflora foetida } \mathrm{L} \text {. } \\
\text { Passifloraceae }\end{array}$ & Sanojhar & Anti-ulcer, antioxidant, diuretic, asthma \& skin diseases. \\
\hline $\begin{array}{l}\text { 42. Piper bettle L. } \\
\text { Piperaceae }\end{array}$ & Pan & $\begin{array}{l}\text { Mouth refreshment; improve digestion, diuretic, } \\
\text { analgesic, cough \& cold. }\end{array}$ \\
\hline $\begin{array}{l}\text { 43. Piper longum L. } \\
\text { Piperaceae }\end{array}$ & Pipli & Cough, digestion, bronchitis, fever \\
\hline $\begin{array}{l}\text { 44. Piper nigrum L. } \\
\text { Piperaceae }\end{array}$ & Kalo marich & Gastric trouble, cough, fever. \\
\hline $\begin{array}{l}\text { 45. Pothos scandens L. } \\
\text { Araceae }\end{array}$ & Money plant & Antioxidant, antipyretic, epilepsy, rheumatism. \\
\hline $\begin{array}{l}\text { 46. Quisqualis indica } \mathrm{L} \text {. } \\
\text { Combrataceae }\end{array}$ & $\begin{array}{l}\text { Madhumalati } \\
\text { / Baja phul }\end{array}$ & Purgative, diarrhoea, fever, rheumatism \& fever. \\
\hline $\begin{array}{l}\text { 47. Smilax aspera } \mathrm{L} \text {. } \\
\text { Smilaceae }\end{array}$ & Kukurdaino & Paralysis, syphilis, diuretic, skin diseases, blood purifier. \\
\hline $\begin{array}{l}\text { 48. Smilax ovalifolia Roxb. } \\
\text { ex D. Don } \\
\text { Smilaceae }\end{array}$ & Ramdatun & $\begin{array}{l}\text { Venereal diseases, rheumatism, abdominal pain, } \\
\text { dysentery, }\end{array}$ \\
\hline $\begin{array}{l}\text { 49. Tinospora cordifloia } \\
\text { (Willd.) Miers. } \\
\text { Menispermiaceae }\end{array}$ & Gurjo/ Giloy & $\begin{array}{l}\text { Anti-diabetic, malarial fever, hepato-protective, } \\
\text { rheumatism, stomach troubles, diuretic, diarrhoea, } \\
\text { dysentery. }\end{array}$ \\
\hline $\begin{array}{l}\text { 50. Trichosanthes dioica } \\
\text { Roxb. } \\
\text { Cucurbitaceae }\end{array}$ & Parwal & Febrifuge, laxative, refrigerant. \\
\hline $\begin{array}{l}\text { 51. Trichosanthes anguina } \mathrm{L} \text {. } \\
\text { Cucurbitaceae }\end{array}$ & Chichinda & $\begin{array}{l}\text { Purgative, anthelmintic, emetic, refrigerant, syphilis \& } \\
\text { verminopsis. }\end{array}$ \\
\hline
\end{tabular}

\section{ACKNOWLEDGEMENT}

Authors are thankful to the peoples of Palpa district who actively participated and shared valuable information in the field; without their active participation, this research would not have been completed. The first author was also thankful to the National Herbarium and Plant Laboratories, Godavari (KATH), Nepal and Herbarium of Tribhuvan University, Butwal Multiple Campus, Butwal for allowing me to consult herbaria and for other facilities.

\section{REFERENSCES}

Bailey LH (1949) Manual of Cultivated Plants. The Mac Millan Company, New York.

Chaudhary RP (1998) Biodiversity in Nepal: Status and conservation. S. Devi, Saharanpur (U.P.) India and Tecpress Books, Bangkok, Thailand.

CBS (2011) Population Census. Central Bureau of Statistics, Ramshah path, Thapathali, Kathmandu Nepal. DDC (2009) District Profile of Palpa. District Development Committee, Palpa, Government of Nepal.

DCEP (2016) District Climate and Energy Plan for Palpa District. Alternative Energy Promotion Centre, National Rural and Renewable Energy Programme, Khumaltar Height, Khumaltar, Nepal den Dubbelden KC \& Oosterbeek B (1995) The availability of external support effects allocation patterns and morphology of herbaceous climbing plants. Functional Ecology 9: 628-634.

DMP (1969) Flora of Phulchoki and Godawari. Bulletin of the Department of Medicinal Plant, Nepal No. 2. Kathmandu, Nepal.

DMP (1970) Medicinal Plants of Nepal. Bulletin of the Department of Medicinal Plant, Nepal No. 3. Kathmandu, www.tropicalplantresearch.com 
Nepal.

DMP (1984) Medicinal Plants of Nepal (Supplement Volume). Bulletin of the Department of Medicinal Plant, Nepal No. 10. Kathmandu, Nepal.

DMP (1986) Flora of Kathmandu Valley. Bulletin of the Department of Medicinal Plant, Nepal No. 11. Kathmandu, Nepal.

Gentry AH (1991) The distribution and evolution of climbing plants. In: Putz FE \& Mooney HA (eds) The Biology of Vines. Cambridge University Press, Cambridge, pp. 3-51.

Hara H, Stearn WT \& Williams LHJ (1978) An Enumeration of the Flowering Plants of Nepal. Vol. 1. Trustees of British Museum (Natural History), London.

Hara H \& Williams LHJ (1979) An Enumeration of the Flowering Plants of Nepal. Vol. 2. Trustees of British Museum (Natural History) London.

Hara H, Charter AO \& Williams LHJ (1982) An Enumeration of the Flowering Plants of Nepal. Vol. 3. Trustees of British Museum (Natural History), London.

Hooker JD (ed) (1872-1897) The flora of British India, Vols. 1-7. L. Reeve and Co. London.

Isnard S, Rowe NP \& Speck T (2009) Moving with climbing plants from Charles Darwins time into $21^{\text {st }}$ century, Darwin Bicentrial Special Invited Paper, University of California, American Journal of Botany 96 : 1205-1221.

Jongkind CCH \& Hawthrone WD (2005) A Botanical Synopsis of Lians and other forest climbers. In: Bongers F, Parren MPE \& Trare D (Eds) Forest climbing Plants of west Africa, Diversity, Ecology and Management. CAB International, Walligford, Oxfordshire, U.K., pp. 19-39.

Kelly DL (1985) Epiphytes and climbers of a Jamaican Rain Forest: Vertical distribution, life forms and life histories. Journal of Biogeography 12: 223-241.

Mahato RB (1998) Notes on some plants of ethnobotanical importance from Palpa district. Tribhuvan University Journal 21(1): 71-76.

Mahato RB \& Chaudhary RP (2003) Ethnomedicinal study and Antibacterial activities of selected plants of Palpa district, Nepal. Scientific World 2: 38-45.

Manandhar NP (2002) Plants and People of Nepal. Timber Press, Portland, Oregon, USA.

Martin G (1995) Ethnobotany: A Methods manual. Chapman and Hall. London.

Mc Crackers IJ \& Shrestha ML (1992) Field Manual for community and private forestry in Nepal. Part I. Common Tree species. Field Document No. 19 Community Forestry Development Project (Phase II) HMG / UNDP / FAO.

Polunin O \& Stainton A (1984) Flowers of the Himalayas. Oxford University Press, London.

Press JR, Shrestha KK \& Sutton DA (2000) Annotated Checklist of the Flowering Plants of Nepal. The Natural History Museum London, and Central Department of Botany, Tribhuvan University, Nepal.

Richards P (1952) The Tropical Rain Forest. Cambridge University Press, Cambridge.

Schimper AFW (1903) Plant-Geography upon A Physiological Basis. Clarendon Press Oxford. [English Translation by (rev) Fisher WR, (ed) Groom P \& Balfour IB].

Schnitzer SA \& Bongers F (2002) The ecology of lians and their role in forests. Trends in Ecology \& Evolution 17: $223-230$.

Shrestha P (1985) Contribution to the Ethnobotany of the Palpa Area. Contribution to the Nepalese studies, CNAS, Tribhuvan University 12(2): 63-74.

Singh AG, Gautam LP \& Tewari DD (2011) Folk Uses of some medicinal plants of Dobhan VDC of Palpa district, Western Nepal. Journal of Phytology 3(8): 62-67.

Singh S (2016) Ethnobotanical study of some climbers of Parsa district forest of Nepal. Journal of Medicinal Plants Studies 4(4): 06-10

Stainton A (1997) Flowers of the Himalaya (A Supplement). Oxford University Press, Oxford.

Swaine MD, Hawthrone WD, Bongers F \& Aceves TM (2005) Climbing Plants in Ghananian Forest. In: Bongers F, Parren MPE \& Trare D (eds) Forest climbing Plants of west Africa, Diversity, Ecology and Management. CAB International, Walligford, Oxfordshire, U.K., pp. 19-39. 